\title{
The effects of anxiety on motor-steadiness in competitive and noncompetitive conditions
}

\author{
GLE N M. VAUGHT AND STEVEN E. NEWMAN 1 \\ $A L B I O N$ COLLEGE
}

Twenty HA and 20 LA Ss were selected from 101 males who had taken the MAS. The Ss were further divided into competitive and noncompetitive subgroups and administered a simple motor steadiness test. It was found that LA Ss made fewer errors than HA Ss in the steadiness test and that competition exacerbated performance differences between $H A$ and LASs.

Over the years, a number of scales have been developed for the purpose of measuring "manifest" anxiety. Of these scales, Taylor's (1953) MAS seems to have generated the most controversy. Much of the ensuing controversy has to do with whether or not the MAS measures a constant "level of internal anxiety or emotionality, " or whether it measures susceptibility to "anxiety" provoking stimuli. In either case, it should follow that people with high MA scores would differ from low scorers in a variety of performance measures. The literature, however, does not support this contention; rather, such relationships have been less than conclusive.

The present study sought to investigate the relationship between extreme MAS scorers and performance in a simple motor steadiness test. Wiggins, Brokaw, Heckel, \& Saltzberg (1962) reported no differences between high- and low-anxiety groups in a similar motor steadiness test; however, they did not employ the MAS. In addition, the present study attempted to determine the influence of "competition" on extreme MAS scoreres. It was thought that regardless of the theoretical rationale underlying the MAS competition should exacerbate performance differences between high- and low-anxiety Ss. More specifically, since the MAS presumably measures a constant level of anxiety or susceptibility to anxiety provoking stimuli, it is anticipated that competition will have a greater negative effect on high anxious Ss in the motor steadiness task than on low scorers.

Method

The Ss, 40 males, were introductory psychology students from a large midwestern university, unaffiliated with the author's institution. The MAS was administered to the Ss during their regular class period and told that this was the first of a two-part experiment. Following MAS testing, all female scores were discarded. From 101 males tested, $20 \mathrm{Ss}$ were selected from the high scores (from 19-32) end of the distribution and 20 from the low end (scores from 4-12). Each :-lentity group was further divided into subgroups of 10 and randomly assigned to competitive-noncompetitive conditions ( 2 by 2 factorial design).
The Ss were individually contacted over a three week period and administered the motor steadiness test. The apparatus consisted of a pencil stylus with a $3 \mathrm{~mm}$ hole arranged in a multiholed steadiness box from the Marietta Equipment Company, Marietta, Ohio. A timer automatically measured the intervals between trials and a second timer recorded time in contact with the edge of the apparatus to the hundredth of a second. Each $S$ was given nine trials but only the mean of the five middle trials was used in the data analysis. Hopefully, this procedure controlled for possible "warm-up" effects as well as the "end-spurt" difficulties.

In order to increase the prestige value of the experimenter, the instructor of the participating introductory class, introduced the junior author as a representative from a mythical testing service. Furthermore, all data sheets, instruction sheets, and signs seen by the Ss were labeled with the fictitious testing service. A standard set of general instructions was read to all groups; however, the competitive groups received one set of instructions and the noncompetitive groups another.

The competitive groups were told that they had been selected as prospective candidates and that in part their selection had been based on their performance on the first part of this study, and that the second part would contribute to whether or not they were to continue to be candidates. The Ss were never told what they had been chosen for until all data had been collected. In addition, during the course of the steadiness test, each $\mathrm{S}$ was told that his scores were well below the scores of others who had participated in this program.

In contrast, the noncompetitive groups were told that this was a new experiment and that the experimenter was simply trying to devise norms for this task. They were also told that individual scores were really not very important.

\section{Results and Discussion}

The data were collected and analyzed via a 2 by 2 analysis of variance. There was a highly significant difference between HA and LA Ss in motor steadiness $(F=10.14, p<.01)$. The HA Ss were much poorer in the motor steadiness test than the LA Ss. There were no significant differences for competition. The interaction between anxiety and competition was significant ( $F=$ $10.56, p<.01)$. Figure 1 shows this interaction. Duncan's multiple-range test (Edwards, 1960) was used to compare the individual mean scores. A significant difference was found between HA and LA Ss in the com- 


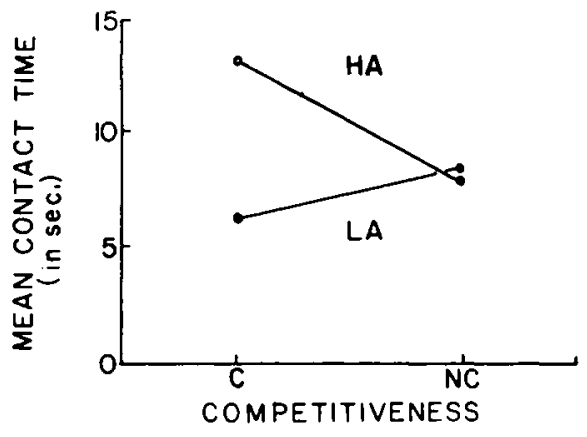

Fig. 1. HA and LA group steadiness scores. Higher scores indicate poorer performance.

petitive situation $(p<.01)$. Figure 1 highlights this finding and shows the LA Ss making fewer errors in the motor steadiness task. A significant difference was found between HA noncompetitive Ss and HA competitive Ss $(p<.01)$, with fewer errors being made by the HA noncompetitive group. The remaining significant difference was between the LA noncompetitive Ss and HA competitive Ss. There was no significant difference between LA noncompetitive and LA competitive Ss.

It is interesting to note that while there was an overall difference between HA and LA Ss in the motor steadiness task the most potent contribution to that difference showed up in the competitive condition. This finding suggests that the MAS does differentiate among people in certain performance tasks provided some motivating condition is included in the experi- mental design. This might, in part, explain the failure of Wiggins et al (1962) and others who have been unable to predict from anxiety scales to performance in a number of similar tasks. These results also suggest that the MAS measures susceptibility to "anxiety" provoking stimuli apart from a constant "level of internal anxiety or emotionality" conception. Thus competition, viewed as a motivational variable, exacerbated performance differences among $\mathrm{HA}$ and LA Ss in the present study.

There are two additional remarks to be made. First, competition was defined in this study by simply establishing the prestige value of the experimenter and by the use of what might be called "negative social reinforcement." Secondly, all the data were collected at a large university some $\mathbf{5 0}$ miles from the author's institution in order to insure naive Ss and to increase the potency of the competition variable. This may, in part, account for the observed interaction.

\section{References}

Edwards, A. L. Experimental design in psychological research. (2nd ed.) New York: Rinehart, 1960.

Taylor, Janet. A. A personality scale of manifest anxiety. $J$. abnorm. soc. Psychol., 1953, 48, 285-290.

Wiggins, S. L., Brokaw, J. R., heckel, R. V., \& Salzberg, H. C. Manifest anxiety and perceptual motor steadiness. Percept. mot. Skills, 1962, 15, 759-762.

\section{Nore}

1. The authors are indebted to the Psychology Department at Western Michigan University and particularly William Yankee. We also thank David Hogberg and William Simpson of Albion College. 